\title{
Circuit dependence of the diameter of pulsed positive streamers in air
}

\author{
T M P Briels ${ }^{1}, \mathrm{~J} \mathrm{Kos}^{1}, \mathbf{E}$ M van Veldhuizen ${ }^{1}$ and U Ebert $^{1,2}$ \\ ${ }^{1}$ Department of Applied Physics, Technische Universiteit Eindhoven, PO Box 513, 5600MB \\ Eindhoven, The Netherlands, \\ ${ }^{2}$ Centrum voor Wiskunde en Informatica (CWI), PO Box 94079, 1090GB Amsterdam, The \\ Netherlands \\ E-mail: e.m.v.veldhuizen@tue.nl
}

Received 21 July 2006, in final form 24 October 2006

Published 1 December 2006

Online at stacks.iop.org/JPhysD/39/5201

\begin{abstract}
The diameter and branching structure of positive streamers in ambient air are investigated with a fast iCCD camera. We use different pulsed power circuits and find that they generate different spatial streamer structures. The electrodes have a point-plane geometry and a distance of 40 or $80 \mathrm{~mm}$, and the peak voltages over the discharge gap are up to $60 \mathrm{kV}$. Depending on circuit and peak voltage, we observe streamers with diameters varying gradually between 0.2 and $2.5 \mathrm{~mm}$. The streamer velocity increases with the diameter, ranging from 0.07 to $1.5 \mathrm{~mm} \mathrm{~ns}^{-1}$, while the current density within the streamers stays almost constant. The thicker streamers extend much further before they branch than the thinner ones. The pulsed power supplies are a switched capacitor supply with an internal resistance of $1 \mathrm{k} \Omega$ and a transmission line transformer supply with an impedance of $200 \Omega$; additional resistors change the impedance as well as the voltage rise time in the case of the capacitor supply. We observe that short rise times and low impedance create thick streamers close to the pointed electrode, while a longer rise time as well as a higher impedance create thinner streamers at the same peak voltage over the discharge.
\end{abstract}

\section{Introduction}

Electric breakdown in gases over large distances occurs in several stages. The first stage is the build up of an avalanche. Then, when space charge starts to influence the applied field, the streamer discharge creates a weakly ionized channel. At very high fields this streamer branches. As realized already more than half a century ago [1,2], streamers are difficult to visualize and to describe, due to their rapidity as well as due to their internal multiscale nature [3]. The aim of the present paper is, in particular, to clarify the experimental findings on different streamer patterns, diameters and velocities, and their relation to the external electric circuit.

A variety of streamer diameters under different experimental conditions has been reported. Photographic observations were made 40 years ago by Waters and Jones for a voltage of $270 \mathrm{kV}$ over a $2 \mathrm{~m}$ gap. They show a streamer with a diameter near the anode of roughly up to $20 \mathrm{~mm}$, decreasing further on in the gap to $\sim 2 \mathrm{~mm}$ [4]. On such photographs, however, one easily overestimates the size because the centre of the channel can be overexposed. The experimental conditions of [4] are far from the small gaps that are studied nowadays for pulsed corona applications [5]. On the other hand, the photos in [4] show that a pulse with a relatively long voltage rise time of the order of $1 \mu \mathrm{s}$ can create thick and thin streamer channels and pronounced branching.

Corona streamers created by pulsed positive voltages have been studied mainly in small gaps. Diameters reported in the literature are e.g. $40 \mu \mathrm{m}$ in pure oxygen at 0.5 bar in a gap of $1 \mathrm{~cm}$ and a voltage of about $11 \mathrm{kV}$ [6]. Values obtained for streamers in air are e.g. $0.2 \mathrm{~mm}$ in a wire-plane gap of $35 \mathrm{~mm}$ at $30 \mathrm{kV}$ [7] and $0.5 \mathrm{~mm}$ in a point-plane gap of $20 \mathrm{~mm}$ at $25 \mathrm{kV}$ [8]. In larger gaps limited information is available. Diameters of $10 \mathrm{~mm}$ in a wire-cylinder discharge of $290 \mathrm{~mm}$ diameter using a voltage pulse of $140 \mathrm{kV}$ are reported in [9]. A recent publication [10] shows a very abrupt change from 4 to $\leqslant 0.5 \mathrm{~mm}$ in a wire-plane discharge of $35 \mathrm{~mm}$ with a short voltage pulse with a maximum of $45 \mathrm{kV}$; the thin streamers 
probably occured after the voltage had collapsed. We will show below that errors in diameter measurements can occur not only due to photographic overexposure, but also due to the insufficient resolution of CCD cameras. On the other hand, they will not explain diameters varying by two to three orders of magnitude at normal pressure.

High altitude discharge phenomena also show interesting effects. They have become a subject of study since their discovery in 1989 (see e.g. [11,12]). These transient luminous events are referred to as sprites, elves, blue jets, etc. Sprites, in particular, are thought to be a type of streamer discharges and their larger diameter of up to $100 \mathrm{~m} \mathrm{[13]} \mathrm{corresponds} \mathrm{to}$ the much lower air density at high altitudes. This scaling with air density follows from simple streamer models taking only impact ionization into account [3] and relates experiments at low or high pressure to each other the results are somewhat modified by photoionization [14-16].

The question of the streamer diameter has also played a classical role in streamer theory. Dawson and Winn [17] were the first to perform so-called 1.5D dynamical streamer computations that used a constant value for this diameter. A value of $60 \mu \mathrm{m}$ was thought to be an optimal value at the time. Later a fixed streamer diameter was predicted by minimization arguments by D'yakonov and Kachorovskii [18] and Simakov and Raizer [19] (for a discussion see [3]). In 2D computations the diameter is not an input, but a result of the computation. Recent computations in gaps longer than $5 \mathrm{~mm}$ $[15,16,20-24]$ show that the diameter of both positive and negative single streamers can depend on the applied voltage. Streamer diameters up to $16 \mathrm{~mm}$ are reported in [21] in a pointplane gap.

Simulations in a homogeneous field show that streamers can expand in overvolted gaps [15,16,20,23-26]. Calculations and measurements are still difficult to compare as they use different voltage pulse parameters and initial and boundary conditions. A recent attempt to compare measurements and calculations for single positive streamers in air shows that this is still not a straightforward task [8].

In the present paper, our goal is to set a basis for such a comparison by performing well-defined experiments that determine how experimental streamer properties and, in particular, the optical diameter depend on applied voltage and gap length. We investigate positive streamers in a point-plane geometry in ambient air. The experiments are performed in the same experimental setup, but with different power supplies. We find that the applied voltage is a key parameter but that the internal resistance and the rise time of the power supply have a distinct influence as well.

The paper is organized as follows: in section 2, the experimental setup and diagnostics are described. Section 3 contains the experimental results and section 4 further discussion and conclusion.

\section{Experimental setup and diagnostics}

\subsection{The two pulsed power supplies used}

Two different power supplies are used to generate pulsed positive streamers. In both supplies first a capacitor is charged and then a switch is closed. However, the consecutive discharging of this capacitor over the needle-plate gap differs.

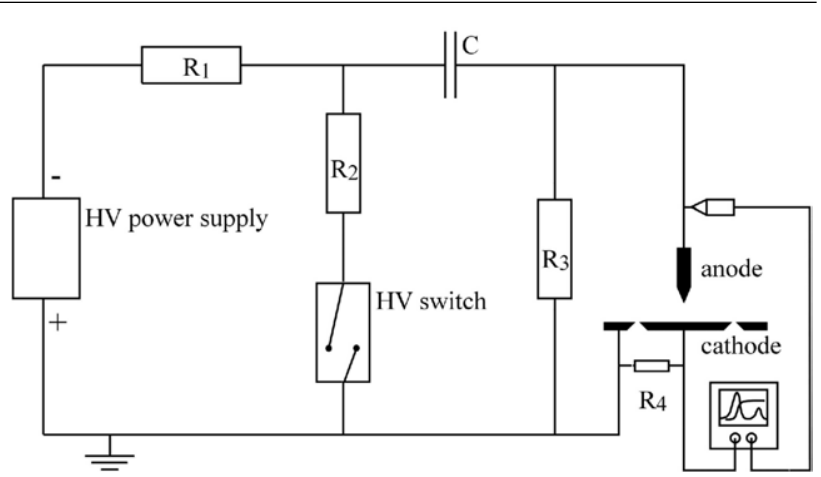

Figure 1. The electric circuit to be called C-supply in this paper.

The first power supply (called C-supply) is sketched in figure 1; it is similar to the supply used previously [27]. The capacitor $\mathrm{C}(250 \mathrm{pF})$ is charged negatively through the resistor $R_{1}$ (25 M $\Omega$ ). Closing the homemade, triggered spark gap puts the potential on the needle-plate electrode gap, with the negative polarity applied to the plate. In this way a positive corona is emitted from the needle. The resistor $R_{2}$ can be used to limit the current through the gap and the switch; its value is either 0 or $1 \mathrm{k} \Omega$. Resistor $R_{3}$ determines the duration of the voltage pulse and is $4 \mathrm{k} \Omega$ in a $40 \mathrm{~mm}$ gap and $25 \mathrm{M} \Omega$ in a $80 \mathrm{~mm}$ gap. A resistive-capacitive divider (Tektronix P6015) is used to measure the voltage at the anode. The voltage rise time depends also on the series resistor $R_{2}$. The current through the corona gap is obtained from the voltage across a series resistor $R_{4}$ between cathode and ground. The value of $R_{4}$ is $2.75 \Omega$. The outer ring across the cathode ensures a welldefined, low stray capacity and therefore a fast rise time of the current measurement [28]. All signals are digitized using 0.2 ns sampling time (LeCroy Waverunner 6100A). The energy is determined as the time integral over voltage times current, after the capacitive part of the total current is subtracted [27]. Noise is filtered out of the signals before integration [28].

Figure 2(a)-(c) shows the measured voltage, current and energy of the discharge when the dc voltage is set to $V_{\mathrm{DC}}=$ $60 \mathrm{kV}$. The resistors $R_{2}$ and $R_{3}$ here are 1 and $4 \mathrm{k} \Omega$. This leads to a rise time of the voltage pulse of $\sim 60 \mathrm{~ns}$, while when using $R_{2}=0 \mathrm{k} \Omega$ the rise time becomes $\sim 30 \mathrm{~ns}$ as is discussed in more detail in section 4.1. The decay of the voltage is controlled by resistor $R_{3}$. In the $40 \mathrm{~mm}$ gap a rather low value of $4 \mathrm{k} \Omega$ is chosen to limit the time during which the voltage is at its maximum. This prevents the transition of the corona discharge into a spark. It also means that the voltage $V_{\mathrm{DC}}$ applied on the capacitor is not reached on the gap since the capacitor rapidly starts to discharge through resistor $R_{3}$. With the dc voltage set to $60 \mathrm{kV}$, the maximum or peak $V_{\mathrm{P}}$ of the voltage pulse on the discharge gap is here $42 \mathrm{kV}$; in general, it will depend on the value of $R_{3}$ and the discharge current. The measured current consists of a capacitive part and a discharge part. The geometric capacity of the current measurement system is determined to be $350 \mathrm{fF}$ at $3 \mathrm{kV}$. At $42 \mathrm{kV}$ this capacitive part has a negligible influence on the energy content of the corona pulse which is $\sim 6 \mathrm{~mJ}$ in the case of figure 2.

We estimate that roughly $50 \%$ of this energy is used for the primary streamer propagation. This estimate is based on the assumption that the maximum current is achieved when the 


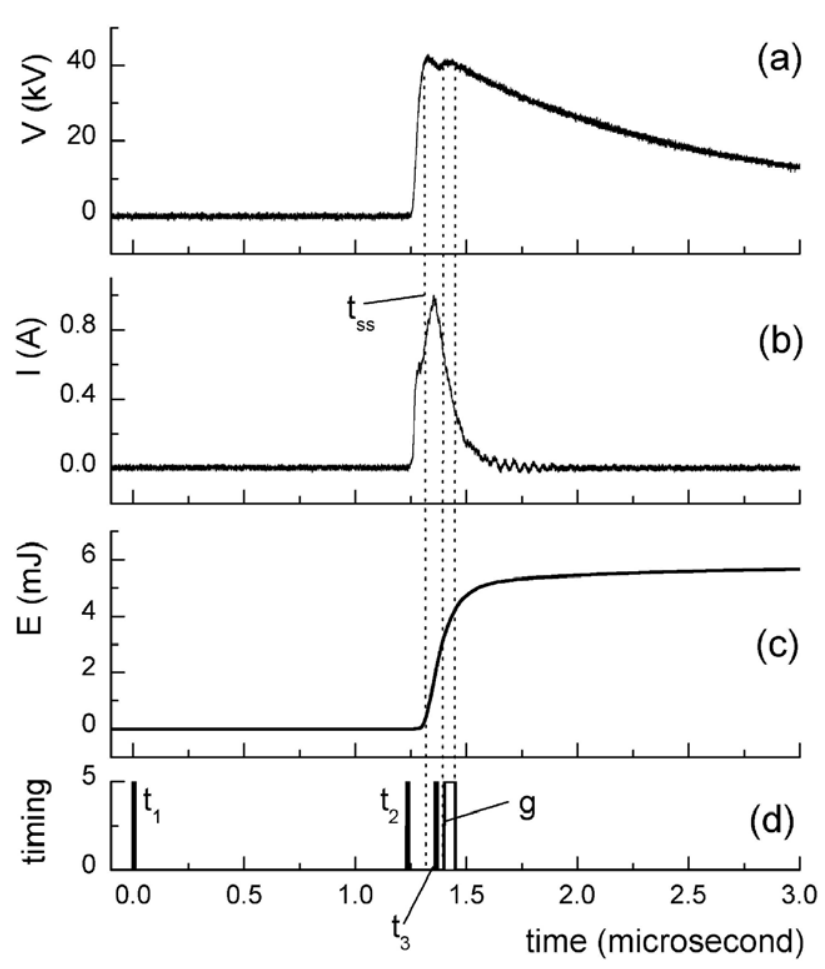

Figure 2. Evolution of voltage (a), current $(b)$ and energy in the corona gap $(c)$ obtained with the C-supply and a charging voltage of $V_{\mathrm{DC}}=60 \mathrm{kV}, R_{2}=1 \mathrm{k} \Omega$ and $R_{3}=4 \mathrm{k} \Omega$. The peak current and voltage on the $40 \mathrm{~mm}$ gap are $I_{\mathrm{P}}=1 \mathrm{~A}$ and $V_{\mathrm{P}}=42 \mathrm{kV}$. A total time interval of $3 \mu \mathrm{s}$ is shown. The voltage rise time is $\sim 60 \mathrm{~ns}$ and the decay time is $5 \mu \mathrm{s}$. In $(d)$ the timing of the measurement is indicated as described in the text: $t_{1}$ is the initial starting pulse for the trigger unit of the complete experiment, $t_{2}$ is the actual trigger for the high voltage pulse, $t_{3}$ is the trigger for the camera with an adjustable delay, $t_{\mathrm{ss}}$ is the moment at which the streamers start (this time can jitter considerably), and $g$ is the actual opening gate of the CCD camera; here it is $50 \mathrm{~ns}$.

primary streamers reach the cathode. Figure 2 shows that at this point the energy is halfway, i.e. $3 \mathrm{~mJ}$ of the total $6 \mathrm{~mJ}$. This fraction, however, very strongly depends on the pulse duration and the gas composition. It is beyond the scope of the present paper to treat this subject in detail. The other half of the energy is consumed after the streamers reach the cathode, in the socalled secondary streamer. In the figure, the corona current reaches its maximum $I_{\mathrm{P}}$ at time $t=1.4 \mu \mathrm{s}$. At that moment, a small dip can be seen in the voltage. This voltage drop of about $2 \mathrm{kV}$ is in agreement with the voltage drop across $R_{2}$ and an internal resistance in the supply at the measured current of $1 \mathrm{~A}$. The peak current $I_{\mathrm{P}}$ is an almost pure particle current, as the displacement current is negligible near the maximum of the voltage.

Figure $2(d)$ shows the timing diagram for the control of the CCD camera. At $t_{1}$, a function generator creates the starting pulse. This pulse is mostly a single shot, manual pulse but it can also be one from a steady pulse train. The pulse goes to the trigger unit of the spark gap. The trigger circuit creates an optical signal which enters through a fibre into the shielded high voltage box where it is converted and amplified to an $8 \mathrm{kV}$ pulse. The total delay of the trigger unit $t_{2}$ has a fixed value of $1.2 \mu \mathrm{s}$. The spark gap follows $10-20 \mathrm{~ns}$ after this $8 \mathrm{kV}$ pulse and then the high voltage pulse starts. The start of corona

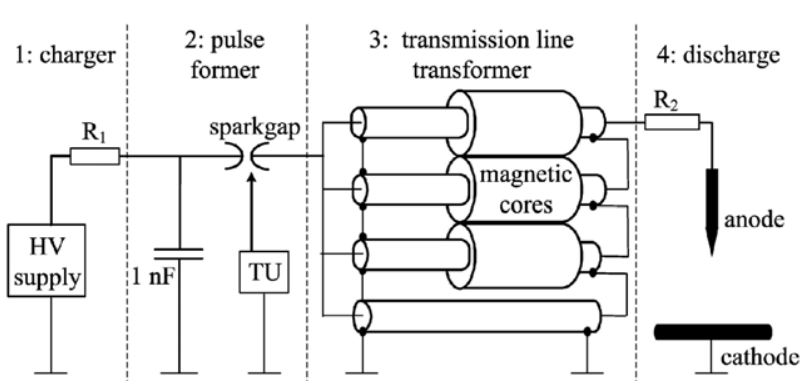

Figure 3. The electric circuit to be called TLT-supply in this paper Parts 1-4 are explained in the text. TU: trigger unit.

streamers is at $t_{\mathrm{ss}}$, the value of $t_{\mathrm{ss}}-t_{2}$ strongly depends on the voltage level and rise time. In figure 2 it is about $50 \mathrm{~ns}$, but it can be much longer if the peak voltage is only just above inception. In that case the jitter of $t_{\mathrm{ss}}-t_{2}$ can be up to several microseconds. The signal $t_{1}$ has simultaneously been sent to a delay generator that gives a pulse to the CCD camera at $t_{3}$. The camera opens its gate $35 \mathrm{~ns}$ after $t_{3}$ with an adjustable duration or 'gate' $g$. In the figure the value of $g$ is set to $50 \mathrm{~ns}$, the camera allows settings from 2 ns to 'infinite'. With low discharge jitter, $t_{3}$ and $\mathrm{g}$ can be chosen in such a way that the streamers cross only a part of the gap during the gate time of the camera, an example of such a snapshot is shown in figure $8(a)$. If the jitter is high, it is unlikely that the camera observes the streamers with a short gate time $g$. In that case photos are taken mostly with very long gate times, as in figure $6(d)$.

The second power supply (called TLT-supply) is shown in figure 3 . Here again a capacitor $(1000 \mathrm{pF})$ is charged (part 1 of the figure), but in this case to a positive polarity. It discharges when the spark gap is closed (part 2) via a transmission line transformer (part 3) over the electrode gap (part 4 of the figure). This supply in principle transforms the voltage up by a factor of 4. Note that this supply is not inverting because the positions of capacitor and spark gap are exchanged in comparison to figure 1 . The TLT provides $12.5 \Omega$ load impedance to the pulse former, but $200 \Omega$ to the discharge [29]. Therefore it is able to create high current pulses with better matching than a $\mathrm{C}$-supply. Voltage and current are measured here with a Tektronix high voltage probe (P6015) and a Pearson current monitor (6585). The magnetic cores are inserted to damp reflections when the load is not well matched to the source.

Typical waveforms of voltage, current and energy are shown in figure 4. A series resistor of $R_{2}=0$ or $1 \mathrm{k} \Omega$ is used again to probe its effect on the discharge. The voltage rise time is $\sim 25 \mathrm{~ns}$, and it is independent of the series resistor $R_{2}$. Figure 4 shows a current of $\sim 12 \mathrm{~A}$ with $R_{2}=1 \mathrm{k} \Omega$ obtained at $14 \mathrm{kV}$ charging voltage. When taking an additional voltage drop of $12 \mathrm{kV}$ across $R_{2}$ into account, this amounts to a transformation ratio of 3.8. In another case with $R_{2}=0$ a peak voltage of $41 \mathrm{kV}$ is obtained at $11.3 \mathrm{kV}$ charging voltage, so the transformation ratio is 3.6. Probably the higher current in this case leads to more losses in the TLT.

The pulse shape appears to be independent of the value of $R_{2}$ for the values used here. The width of the corona pulse is indicated in the figure with two dotted lines that are $70 \mathrm{~ns}$ apart (the first peak is capacitive and the actual corona current peak is $\sim 50 \mathrm{~ns}$ ). The energy content, as shown in figure 4 , is $\sim 15 \mathrm{~mJ}$ up to the second dotted line. The oscillations after $100 \mathrm{~ns}$ show 


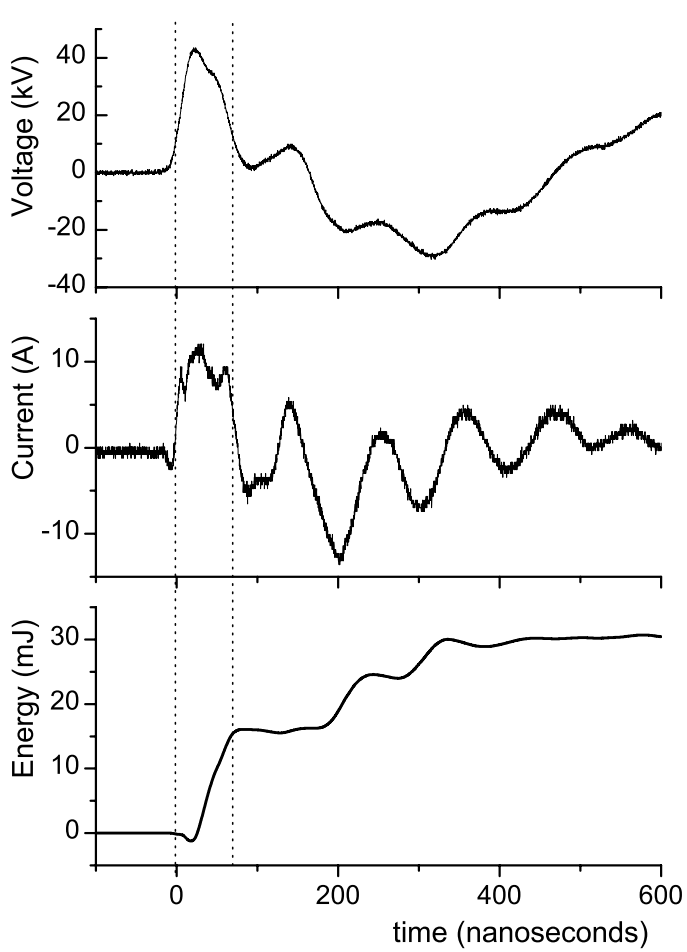

Figure 4. Evolution of voltage, current and energy on the TLT-supply with a charging voltage of $14 \mathrm{kV}$ in an $80 \mathrm{~mm}$ gap with $R_{2}=1 \mathrm{k} \Omega$. The peak current and voltage are $I_{\mathrm{P}}=12 \mathrm{~A}$ and $V_{\mathrm{P}}=40 \mathrm{kV}$. The voltage rise time is $\sim 24 \mathrm{~ns}$ and the corona pulse duration is $\sim 50 \mathrm{~ns}$.

that the impedance of the corona discharge does not match the power supply. The energy from these oscillations anyway does not contribute to the primary streamer propagation. The matching of TLT-supplies to a corona discharge can be almost perfect in long wire-cylinder discharges at very high currents [29].

\subsection{Diagnostic procedure}

In all measurements reported here, a point-plane electrode configuration is used, and the distance between the electrodes is adjustable. The anode tip is made of thoriated tungsten and has a radius of $\sim 15 \mu \mathrm{m}$. The round cathode inner plate is $100 \mathrm{~mm}$ in diameter and the outer ring of the divided cathode has an outer diameter of $180 \mathrm{~mm}$. All measurements are performed in ambient air at normal pressure.

Photographs of the discharge are taken with an intensified CCD camera, a 4QuikE from Stanford Computer Optics with $736 \times 572$ pixels with $8.6 \times 8.3 \mu \mathrm{m}$ pixel size. The camera is sensible in the optical wave length range of $300-800 \mathrm{~nm}$; the figures are actually dominated by emission of the $\mathrm{N}_{2}(C-B)$ transition that has a decay time of about $1 \mathrm{~ns}$. In the figures within this paper, the focal depth is about $2 \mathrm{~cm}$.

The streamer diameter is determined from iCCD photographs such as in figure 5. When measuring the diameter, care is taken that measurements are done on a single streamer at a place without return stroke, multiple streamers, anode glow or 'out-of-focus' effect. To avoid measuring effects of the return stroke, the camera's gate width is chosen in such a way that only the primary streamer during its flight is photographed, i.e. the gate is less than $100 \mathrm{~ns}$. Voltage oscillations as in figure 4 then

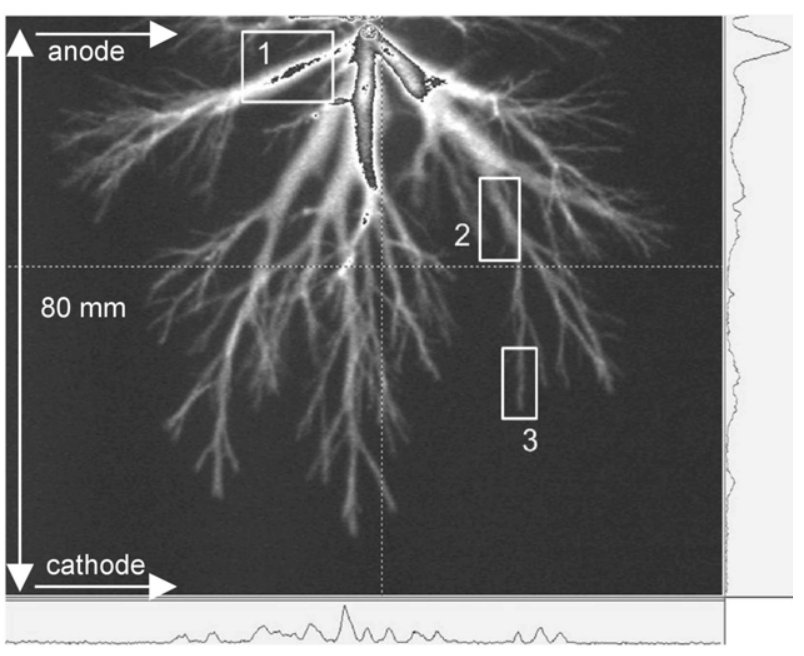

Figure 5. Streamers obtained with the C-supply in an $80 \mathrm{~mm}$ gap with $R_{2}=0, V_{\mathrm{P}}=60 \mathrm{kV}$ and $I_{\mathrm{P}}=10 \mathrm{~A}$. Profile bars at the positions indicated by the dotted lines are shown at the right and at the bottom. The optical gate for this photo is $\sim 80 \mathrm{~ns}$, during this time the streamers have not crossed the gap yet. The boxes indicate very thick (type 1), thick (type 2) and thin (type 3) streamers. For further discussion, we refer to section 4 .

do not influence the images. Occasionally a long integration time of several $\mu$ s is used to show the later evolution after the streamers have reached the cathode. This is done particularly for the C-supply where the voltage decays slowly.

The dotted lines in figure 5 indicate the positions of the cross-sections shown in the profile bars below and beside the picture. Each peak in the profile corresponds to a streamer. The streamer diameter is measured as the full width at half maximum (FWHM) in the profile of the respective peak. At this point, we want to warn the reader that these diameters can deviate from the visual impression of the converted and plotted images; in the plotted images, bright channels will appear wider than faint channels when the FWHM diameter is the same.

\section{Results}

\subsection{The influence of voltage and gap spacing}

Figure 5 shows a typical streamer pattern at atmospheric pressure in a gap of $80 \mathrm{~mm}$ under a voltage pulse of $60 \mathrm{kV}$. Near the anode three streamers with a large diameter can be seen. The clearest one is indicated with a box labelled 1 . The FWHM diameter of this streamer is $\sim 2.5 \mathrm{~mm}$ according to the prescription of section 3. The diameter is more or less constant while the streamers propagate towards the cathode. After $\sim 20-30 \mathrm{~mm}$ these thick streamers branch and several thinner streamers emerge with a diameter of $\sim 1.2 \mathrm{~mm}$. One such streamer is again indicated with a box, labelled 2. Then after shorter travel distances of the order of 5-10 $\mathrm{mm}$, the streamers branch again into even thinner channels. Again, such a thin streamer is indicated by a box and labelled 3 . The streamers from now on will be referred to as type 1,2 or 3 according to the box number shown in figure 5 though we stress that there seems to be a continuous transition between streamers of different diameters; therefore the types should 
Table 1. Diameters of streamers of type 3 in a gap of $80 \mathrm{~mm}$ obtained with different zoom factors.

\begin{tabular}{lll}
\hline Total view $(\mathrm{cm})$ & FWHM width (\# pixels) & Apparent size $(\mu \mathrm{m})$ \\
\hline 8 & $4-5$ & $620-770$ \\
4 & $4-5$ & $290-380$ \\
2 & $5-6$ & $190-230$
\end{tabular}

not be misunderstood as a classification of distinct propagation modes.

Reading the diameter of the type 3 streamers from figure 5 leads to a value of $\sim 0.7 \mathrm{~mm}$. In this situation, however, one pixel of the CCD camera corresponds to $0.15 \mathrm{~mm}$. Taking into account that there is always some cross-talk between adjacent cells of a CCD array, one could suspect that these $0.7 \mathrm{~mm}$ are an overestimation. Indeed, when the CCD camera zooms in into the lower part of the discharge with a factor of 2 or a factor of 4 , at least 4 pixels in a row transverse to the streamer are always illuminated, as summarized in table 1 .

It is clear that the diameter of the thin streamer is broadened due to instrumental effects when a large gap is imaged onto our CCD camera. When the total view of the camera is decreased to $2 \mathrm{~cm}$, additional broadening sets in with now 5-6 pixels being illuminated; this measured result of about $200 \mu \mathrm{m}$ probably approaches the real streamer diameter. Zooming in further gives problems with focal depth and finding a streamer that meets all requirements for a correct determination of the FWHM. This value of $200 \mu \mathrm{m}$ is also close to values reported earlier $[8,27,30]$. The measured diameters of the wider streamers of types 1 and 2 are, within experimental errors, not affected by zooming in.

First observations in a $40 \mathrm{~mm}$ gap using the TLT-supply have been given in [10] and in [3]. When compared with the photographs of e.g. [27], these pictures appear to give the idea of completely different streamer types, that would be distinguished by different propagation modes and separated by phase transitions. At that time the different appearance was essentially attributed to the TLT-supply with its low internal impedance. Here it will be shown that thick streamers with almost no branching also occur with the C-supply at sufficiently high voltage.

Figure 6 shows four streamer patterns in a shorter gap of $40 \mathrm{~mm}$ with a voltage pulse of $54 \mathrm{kV}$ using the C-supply. (Because spark formation had to be prevented, higher voltages could not be explored in this short gap.) Figure 6(a) is taken with a camera gate time $g=4 \mathrm{~ns}$ and such a delay $t_{3}$ that the observed streamers are in the middle of the gap. As shown previously [3,31], the complete streamer does not emit light, but only the actively growing heads of the channels do so. The image shows the path that the streamer heads have crossed within the gate time, hence the velocity can be determined. In figure 6(a), the travelled distance is $\Delta y=6 \mathrm{~mm}$ and the gate time is $g=4 \mathrm{~ns}$; therefore the local velocity at this part of the gap is $1.5 \mathrm{~mm} \mathrm{~ns}^{-1}$. Note that there is some ambiguity in this determination as some streamers propagate towards or away from the camera and are therefore optically shortened. For our velocity measurements, we choose streamers that stay in focus and that we believe to propagate within the image plane.
Figure $6(b)$ is taken with a gate time of the CCD intensifier of $50 \mathrm{~ns}$. The streamers have not yet reached the cathode during this time interval because the gate opened some time before the streamers started. Several thick streamers of type 1 start at the anode; their diameter remains constant or even seems to increase and very few thinner branches just appear. The overall pattern in this photo is quite similar to the pattern of the thickest streamers in figure 5 when the different sizes (40 versus $80 \mathrm{~mm}$ ) of the gaps are taken into account.

Figure $6(c)$ shows the discharge development taken with the same gate time of $50 \mathrm{~ns}$. But due to some jitter in the spark gap and the discharge inception, this picture shows a somewhat later stage of the development than figure $6(b)$. The figure shows that the type 1 streamers can fully bridge the gap within these $50 \mathrm{~ns}$ as also observed in [3] and [10]. A new interesting feature appears; streamers with a diameter similar to those of type 3 in figure 5 start in the neighbourhood of the point. These thin streamers will be called type 4 . They mostly start after the thick streamers have reached the cathode. They seem to start at some surface roughness higher up on the anode or somewhere along an existing streamer path. When the integration time of the camera is longer, the thin streamers cross the whole gap. Figure 6(c) is chosen here particularly because it is not overcrowded with streamers and because it shows how late streamers have emerged from the anode but have not reached the cathode yet. Figure $6(d)$ shows how many thin late streamers cross from anode to cathode when a longer gate time is used. Late streamers of type 4 are not observed when using the TLT-supply because the pulses of this supply are much shorter in time (see table 2).

Another remarkable event is observed in the circle drawn in figure 6(c). A late streamer does not continue its way to the cathode but appears to bend towards an existing streamer path and seems to connect to it. This effect can, of course, not clearly be concluded from 2D photographs of a 3D event, but it is observed on many photos that a streamer bends towards another straight one and precisely stops at the straight streamer and practically never just before or just after. A plausible explanation for this observation is that a 'return stroke' changed the polarity of the early thicker streamer after it connected to the cathode, and that it therefore became electrically attractive for the late streamer. This effect was observed before [10,27]; a similar event, but probably without prior connection to an 'electrode', was recently observed in sprites [32].

Now the behaviour in the wider gap of $80 \mathrm{~mm}$ as in figure 5 is studied. For this gap length, pulses of $60 \mathrm{kV}$ are strong enough to let the streamers bridge the gap. First, figure $7(a)$ shows streamers in an $80 \mathrm{~mm}$ gap at an applied voltage of $40 \mathrm{kV}$ using the TLT-supply. These streamers die out roughly halfway through the gap. They start as thick streamers at the anode with a diameter corresponding to type 2 and branch into type 3 streamers after $\sim 10-40 \mathrm{~mm}$.

In figure $7(b)$ the voltage is increased to $60 \mathrm{kV}$. The gate time of the photograph is taken so short that the streamers have not yet reached the cathode. At the anode two streamers of type 1 diameter can be seen that branch into streamers of type 2 after $\sim 20 \mathrm{~mm}$. The streamer pattern in this figure, after branching into type 2 streamers, is very similar to the streamer pattern in figure 7(a) starting right at the anode. A possible conclusion is that the end of the type 1 streamer in the $60 \mathrm{kV}$ 

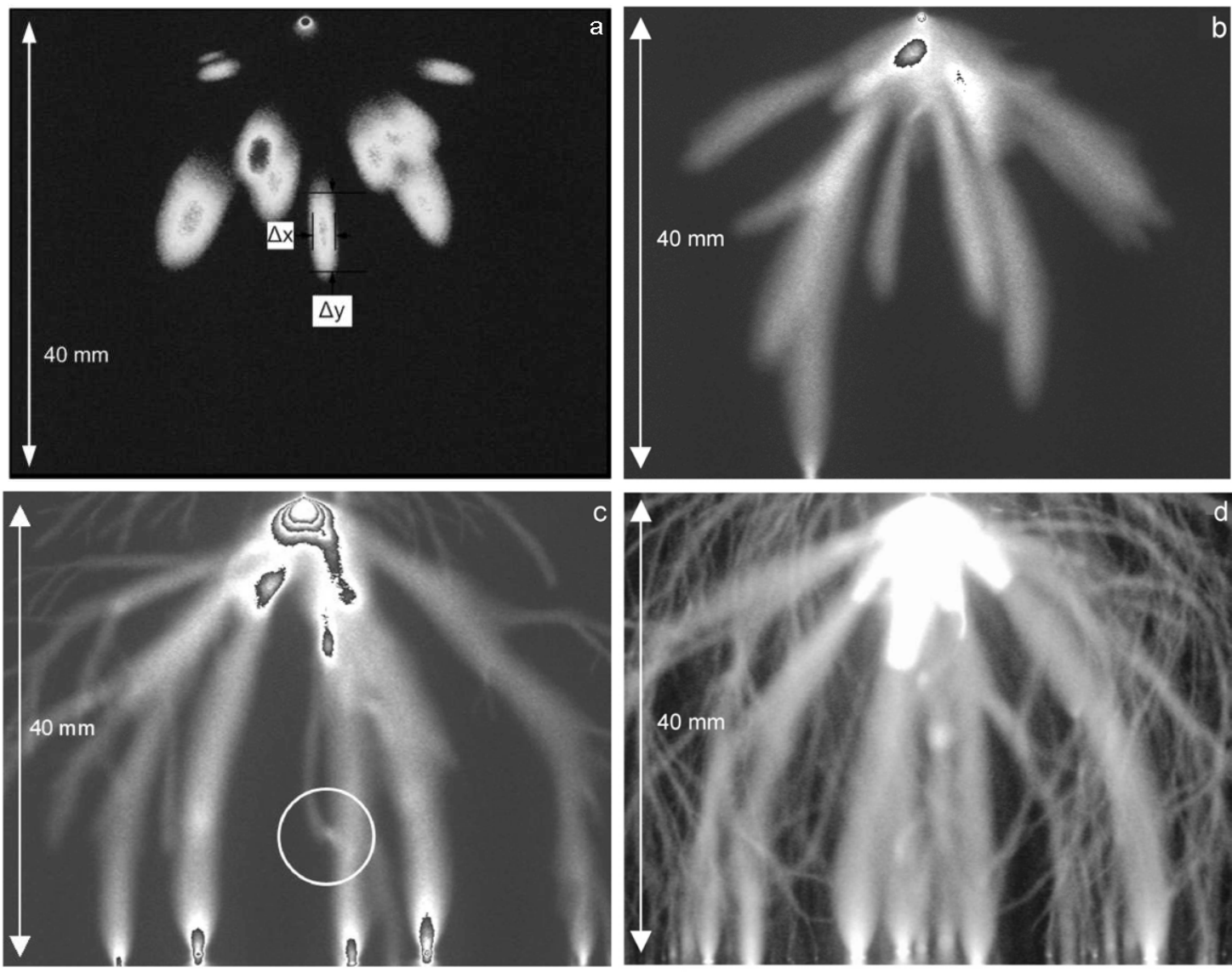

Figure 6. Streamers in a $40 \mathrm{~mm}$ gap exposed to $54 \mathrm{kV}$ with the C-supply. $\left(R_{2}=0, V_{\mathrm{DC}}=60 \mathrm{kV}, V_{\mathrm{P}}=54 \mathrm{kV}\right.$ and $I_{\mathrm{P}}=11 \mathrm{~A}$. $)$ Camera gate time: (a) $4 \mathrm{~ns},(b) 50 \mathrm{~ns},(c)$ also $50 \mathrm{~ns},(d) 1.8 \mu \mathrm{s}$.

Table 2. Electrical properties of the pulses. $V_{\mathrm{DC}}$ is the voltage of the source and $R_{2}$ a resistance in the circuit, see figures 1 and $3 . V_{\mathrm{P}}$ and $I_{\mathrm{P}}$ are the peak voltage and peak current at the discharge. $t_{\mathrm{R}}$ is the voltage rise time, $\mathrm{d} t$ the halfwidth of the current peak and $E$ the integrated energy of the total peak.

\begin{tabular}{lllllclll}
\hline & $\begin{array}{l}\text { gap } \\
(\mathrm{mm})\end{array}$ & $\begin{array}{l}V_{\mathrm{DC}} \\
(\mathrm{kV})\end{array}$ & $\begin{array}{l}R_{2} \\
(\mathrm{k} \Omega)\end{array}$ & $\begin{array}{l}V_{\text {peak }} \\
(\mathrm{kV})\end{array}$ & $\begin{array}{l}I_{\text {peak }} \\
(\mathrm{A})\end{array}$ & $\begin{array}{l}t_{\mathrm{R}} \\
(\mathrm{ns})\end{array}$ & $\begin{array}{l}\mathrm{d} t \\
(\mathrm{~ns})\end{array}$ & $\begin{array}{l}E \\
(\mathrm{~mJ})\end{array}$ \\
\hline C-supply & 40 & 40 & 0 & 40 & 1.7 & 30 & 80 & 6.4 \\
& & $60^{\mathrm{a}}$ & 0 & 54 & 11 & 30 & 70 & 59 \\
& & $60^{\mathrm{a}}$ & 1 & 42 & 1 & 60 & 120 & 5.5 \\
& 80 & 60 & 0 & 60 & $\sim 10^{\mathrm{b}}$ & 27 & & \\
TLT-supply & 40 & 60 & 1 & 57 & $\sim 3^{\mathrm{b}}$ & 51 & & \\
& & 11.3 & 0 & 40 & 22 & 25 & 25 & 19 \\
& & 17.2 & 0 & 60 & 55 & 26 & 35 & 95 \\
& & 11.3 & 0 & 40 & 19 & 24 & 35 & 22 \\
& & 16 & 0 & 60 & 48 & 23 & 25 & 72 \\
& & 14 & 1 & 40 & 13 & 24 & 50 & 17 \\
& & 22 & 1 & 60 & $\sim 20^{\mathrm{b}}$ & 23 & & \\
\hline
\end{tabular}

a The values indicated are measured with $R_{3}=4 \mathrm{k} \Omega$; for the rest

$R_{3}=25 \mathrm{M} \Omega$.

$\mathrm{b}$ The values indicated are an estimate.

discharge plays a similar role as the electrode needle for the $40 \mathrm{kV}$ discharge; it supplies a similar current and voltage for the further streamer evolution.

Figure 7(b) shows frequent streamer branching that creates many type 3 streamers in practically the whole gap, but in the most pronounced way further away from the anode. These thin streamers do reach the cathode, as is observed in pictures with a longer optical gate. However, type 4 streamers have not been observed in the $80 \mathrm{~mm}$ gap, neither with the short pulse of the TLT-supply nor with the C-supply.

\subsection{The influence of the power supply}

The C-supply and the TLT-supply have both been operated with a peak voltage $V_{\mathrm{P}}$ on the gap of 40 and $60 \mathrm{kV}$ and with $R_{2}$ 

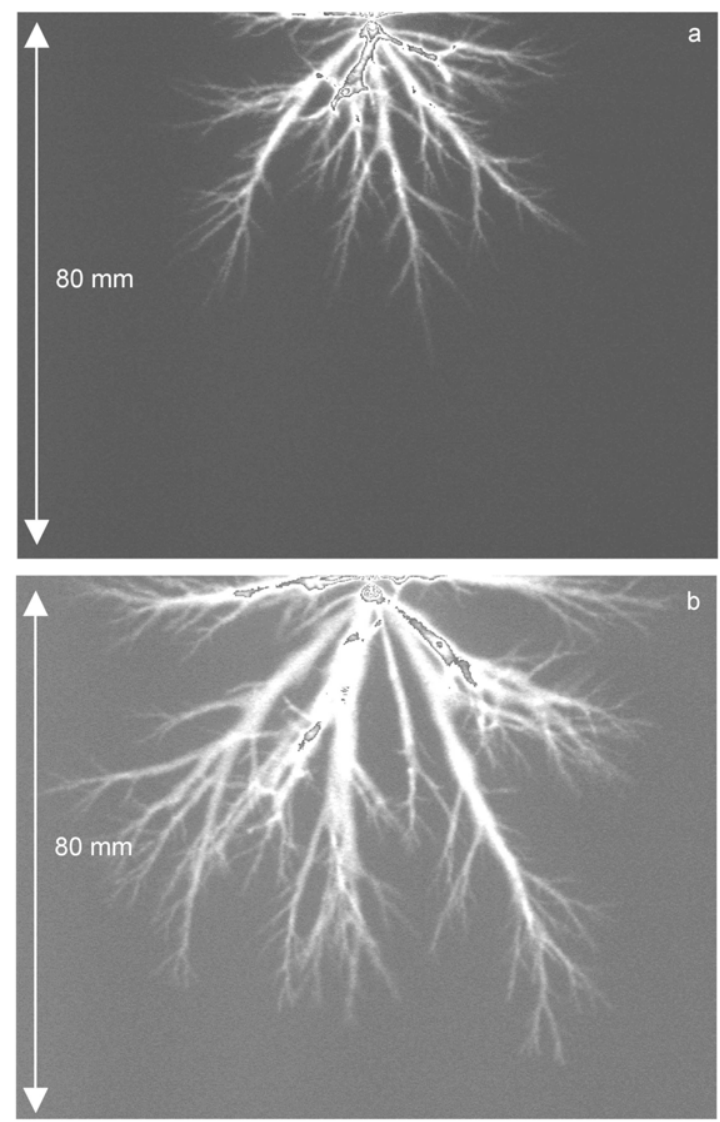

Figure 7. Streamers in an $80 \mathrm{~mm}$ gap with TLT-supply and $R_{2}=0$. (a) $V_{\mathrm{DC}}=11 \mathrm{kV}, V_{\mathrm{P}}=40 \mathrm{kV}, I_{\mathrm{P}}=19 \mathrm{~A}$. (b) $V_{\mathrm{DC}}=16 \mathrm{kV}$, $V_{\mathrm{P}}=60 \mathrm{kV}, I_{\mathrm{P}}=48 \mathrm{~A}$. In figure $(b)$, the voltage pulse of $60 \mathrm{kV}$ is high enough and the pulse duration is long enough for the streamers to bridge the gap, as other observations show. However, the gate width in this particular picture is chosen so short that the primary streamers have not crossed the gap yet during the camera gate time.

set to 0 or $1 \mathrm{k} \Omega$. The maximum of the dc supply is $60 \mathrm{kV}$ so the peak voltage of the pulse is lower if there is a considerable current pulse across $R_{2}$. The parameters of the electrical pulses used in this paper are summarized in table 2 .

Table 2 is not complete because some current measurements were considered to be unreliable due to oscillations. Nevertheless, the table does show several remarkable effects as follows:

1. For a given peak voltage the peak current decreases slightly with increased gap spacing.

2. The series resistor $R_{2}$ of $1 \mathrm{k} \Omega$ reduces the peak current by a factor $\sim 0.1-0.3$ in the case of the C-supply and by a factor $\sim 0.4-0.6$ for the TLT-supply.

3. For $R_{2}=0$ the TLT has a transformation ratio of $3.6 \pm 0.1$ between peak voltage $V_{\mathrm{P}}$ on the corona gap and $V_{\mathrm{DC}}$ of the power source, and for $R_{2}=1 \mathrm{k} \Omega$ this ratio goes down to $2.8 \pm 0.1$. When the voltage across $R_{2}$ is added the ratio becomes $3.7 \pm 0.1$.

4. The rise time of the $\mathrm{C}$-supply depends on $R_{2}$. The rise time of the TLT-supply does not depend on $R_{2}$.

5. The peak currents with the TLT-supply are 5-10 times larger than those with the C-supply for identical pulse voltages $V_{\mathrm{P}}$ and gaps.
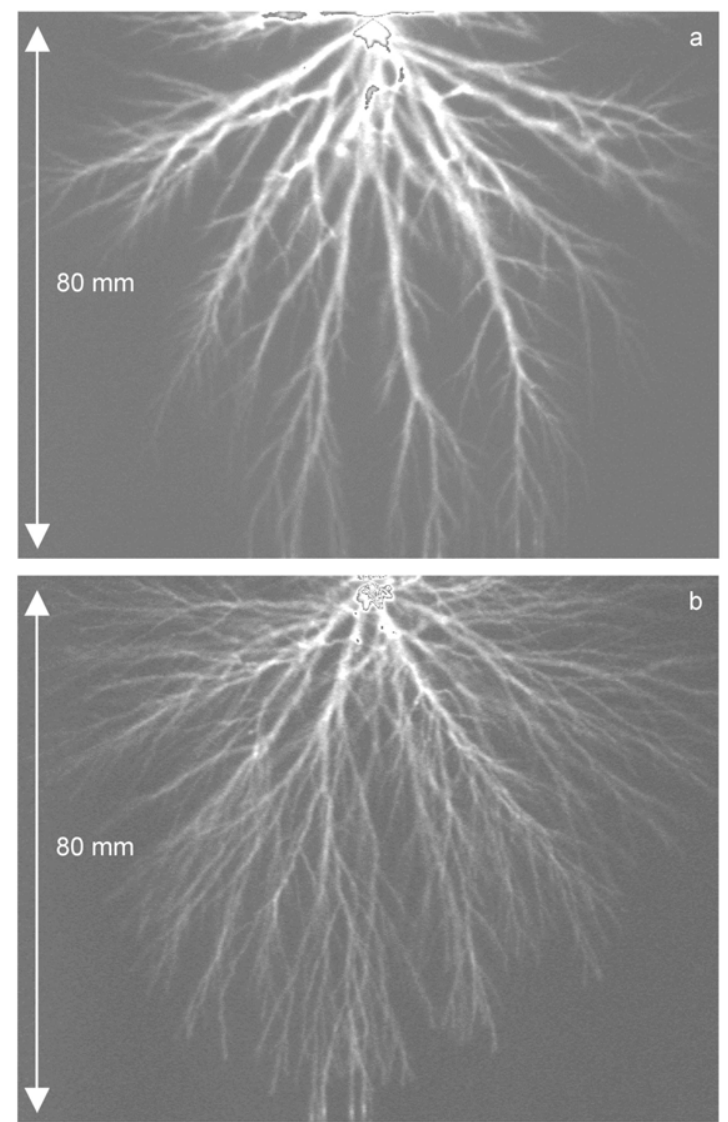

Figure 8. Streamers in an $80 \mathrm{~mm}$ gap with $R_{2}=1 \mathrm{k} \Omega$. (a) TLT-supply with $V_{\mathrm{DC}}=22 \mathrm{kV}, V_{\mathrm{P}}=60 \mathrm{kV}, I_{\mathrm{P}}=20 \mathrm{~A}$, optical gate $50 \mu$ s. (b) C-supply with $V_{\mathrm{DC}}=60 \mathrm{kV}, V_{\mathrm{P}}=57 \mathrm{kV}, I_{\mathrm{P}}=3 \mathrm{~A}$, optical gate $1.4 \mu \mathrm{s}$.

6. The pulse duration of the TLT-supply is shorter.

7. The energies per pulse are a factor of 2 to 3 higher for the TLT-supply. This comparison is troublesome because the incompleteness of the dataset is most obvious here. Further, the longer duration of the pulses of the C-supply contain energy of the secondary streamers and the energy of the TLT-supply pulses can be inaccurate due to the oscillations of the TLT.

Now the branching patterns obtained with both power supplies will be compared. The streamer patterns created with both power supplies are not very different when $R_{2}$ is 0 (see figures 5 and $7(b)$. The main difference is that the thick streamers propagate further in the gap in the case of the TLTsupply. Table 2 shows that there is a difference of a factor $\sim 4$ in current between figures 5 and $7(b)$.

Figure $8(a)$ shows a picture of the $80 \mathrm{~mm}$ gap in the case when the resistor $R_{2}=1 \mathrm{k} \Omega$ is added to the TLT-supply circuit. The current through the discharge decreases from 48 (figure $7(b)$ ) to $\sim 20 \mathrm{~A}$ (for $V_{\mathrm{P}}=60 \mathrm{kV}$ ) while the rise time remains the same. No type 1 or type 4 streamers can be seen. The branching of type 2 streamers into type 3 streamers occurs in figure $8(a)$ closer to the anode than in figure $7(b)$. So, the current and the streamer thickness is limited here by the impedance.

When the resistor $R_{2}$ is added to the C-supply, not only is the maximum current reduced by a factor of 3 in the $80 \mathrm{~mm}$ gap, but the rise time of the voltage pulse also becomes a factor of 2 
Table 3. Streamer velocities obtained in the $80 \mathrm{~mm}$ gap.

\begin{tabular}{|c|c|c|c|c|c|}
\hline & \multirow{2}{*}{$\begin{array}{l}V \\
(\mathrm{kV})\end{array}$} & \multirow{2}{*}{$\begin{array}{l}R_{2} \\
(\mathrm{k} \Omega)\end{array}$} & \multicolumn{3}{|c|}{$\begin{array}{c}\text { Velocity }\left(\mathrm{mm} \mathrm{ns}^{-1}\right) \\
\text { per streamer type }\end{array}$} \\
\hline & & & 1 & 2 & 3 \\
\hline \multirow[t]{4}{*}{ C-supply } & 40 & 0 & - & 0.4 & 0.1 \\
\hline & & 1 & - & - & - \\
\hline & 60 & 0 & 1.5 & 0.5 & - \\
\hline & & 1 & - & 1 & - \\
\hline \multirow[t]{4}{*}{ TLT-supply } & 40 & 0 & - & 0.35 & 0.07 \\
\hline & & 1 & - & - & - \\
\hline & 60 & 0 & 1.5 & 0.4 & - \\
\hline & & 1 & - & 0.5 & $<0.3$ \\
\hline
\end{tabular}

longer. Figure $8(b)$ shows the streamer pattern in this situation (figure 5 shows the case with $R_{2}=0$ ). Also here there are no type 1 and 4 streamers, and the type 2 streamers are even shorter than in figure $8(a)$. The type 3 streamers fill up the larger part of the gap and branch many times.

The rise time of the voltage in the last case becomes comparable to the gap crossing time of the streamers. So what probably happens is that the streamers initiate from the needle before the voltage has reached its maximum; therefore initially they form a pattern of thinner streamers corresponding to this lower voltage. Apparently, the streamer diameter cannot increase substantially during the later evolution, while streamers can branch into thinner streamers. A future theoretical study of electric currents and potentials within the streamer pattern will have to shed more light on this evolution.

\subsection{The velocity of different streamer types}

The streamer velocity at various places in the electrode gap can be obtained from time resolved measurements as demonstrated, e.g. in figure 6(a) for type 1 streamers or in figure 1 of [3] for type 3 streamers. The travelled distance is measured as the FWHM length of the streamer head path and this is divided by the gate width which typically ranges from 4 to $50 \mathrm{~ns}$. This measurement is complicated by several circumstances. First, the spark gap switches have considerable jitter; therefore the time $t_{2}$ in figure $2(d)$ has a jitter of the order of several tens of nanoseconds. So it is a matter of trial and error to obtain a suitable piece of streamer on a photo in a wanted position in the gap. For this reason table 3 could not be completed. A second cause for incompleteness of this table is that not all types of streamers occur under the conditions indicated.

Furthermore, as already said above, there are no really distinguishable streamer types and a streamer can continuously change from type 1 to type 2 and further to type 3 . The table contains measurements on short streamer parts that clearly fall into one particular 'type'. The shortness of the streamers leads to larger errors in the determined velocity. Therefore the velocities in table 3 have errors and should be understood as representing a broad distribution with widths of $20 \%-50 \%$. Late type 4 streamers appear with considerably more jitter in time. This makes it virtually impossible to determine their velocity with the method used here.

Table 3 shows that thick streamers travel faster than thin ones; they also occur closer to the point electrode where the instantaneous local background fields are higher-however, the local background field in the absence of streamers should not be confused with the actual local field when the streamers are present. In ambient air at atmospheric pressure, streamers of type 1 are the fastest with velocities of more than $1 \mathrm{~mm} \mathrm{~ns}^{-1}$. Streamers of type 2 have velocities of $\sim 0.5 \mathrm{~mm} \mathrm{~ns}^{-1}$ and streamers of type 3 have velocities of $\sim 0.1 \mathrm{~mm} \mathrm{~ns}^{-1}$. The velocity range found here is in the same range as in other experiments [7,8] and calculations [7,8,21].

A general trend is that type 2 and type 3 streamers are faster when the applied voltage is higher, but further quantification is not possible at the present stage. For type 1 streamers, the limited amount of data available does not allow conclusions.

\section{Discussion and conclusions}

\subsection{Comparison of power supplies: the role of rise time and internal resistance}

In our point-plane gaps of 40 and $80 \mathrm{~mm}$, with pulse amplitudes in the range of $40-60 \mathrm{kV}$, currents are obtained in the range of 1-55 A (see table 2). This demonstrates the wellknown strongly non-linear relation between peak voltage and peak current of the pulsed corona discharge that in addition are strongly influenced by the different power supplies with their different voltage rise times and internal resistances.

In the C-supply for $V_{\mathrm{DC}}=40 \mathrm{kV}$, the voltage rise time increases from 30 to $60 \mathrm{~ns}$ when the series resistance $R_{2}=$ $1 \mathrm{k} \Omega$ is added. Assuming that this is an RC charging time, the internal resistance of the power supply, $R_{\mathrm{C}}$ also has to be $\sim 1 \mathrm{k} \Omega$. This agrees with a dip of $\sim 2 \mathrm{kV}$ when the peak voltage of $V_{\mathrm{P}}=42 \mathrm{kV}$ as seen in figure 2 ; here the peak current is $I_{\mathrm{P}}=1 \mathrm{~A}$ and the series resistance is $R_{2}=1 \mathrm{k} \Omega$ (see table 2). With $R_{2}=0$ the current almost doubles to $1.7 \mathrm{~A}$. This implies that the discharge adapts to the power supply and changes its internal resistance with almost the same factor of 1.7. So the consequence of adding $R_{2}$ in the C-supply is both a limitation of the current and an increase in the voltage rise time. Under these conditions, the streamers are considerably thinner and carry less current.

The output impedance of the TLT-supply is $200 \Omega$. For a peak voltage $V_{\mathrm{P}}=60 \mathrm{kV}$ in the $80 \mathrm{~mm}$ gap, the peak current is $I_{\mathrm{P}}=48 \mathrm{~A}$ when $R_{2}=0$. This corresponds to an internal loss in the power supply of $48 \mathrm{~A} \times 200 \Omega=9.6 \mathrm{kV}$. For this peak, $17.2 \mathrm{kV}$ charging voltage was used, so ideally the TLTsupply should produce $69 \mathrm{kV}$ with a transformation ratio of 4. Therefore the peak voltage at the gap should be $59.4 \mathrm{kV}$, very close to the measured value of $60 \mathrm{kV}$. With $R_{2}=1 \mathrm{k} \Omega$, the current drops to $\sim 20 \mathrm{~A}$ and a charging voltage of $22 \mathrm{kV}$ is required. This leads to a peak voltage on the gap of $4 \times 22 \mathrm{kV}-(1 \mathrm{k} \Omega+200 \Omega) \times 20 \mathrm{~A}=64 \mathrm{kV}$, again close to the measured value of $60 \mathrm{kV}$. For the TLT-supply, the resistor $R_{2}$ limits the current, but has no influence on the voltage rise time.

The hypothesis that the internal resistance of the power supply determines the streamer pattern when peak voltage and rise time are identical, can be tested by comparing experiments with the C-supply and $R_{2}=0$ to experiments with the TLTsupply and $R_{2}=1 \mathrm{k} \Omega$. This is because the C-supply has an internal resistance of $\sim 1 \mathrm{k} \Omega$ as derived from its change in rise time while the TLT-supply with $R_{2}$ added has $1.2 \mathrm{k} \Omega$ resistance 
in total. Indeed, when applying these two power supplies to the $80 \mathrm{~mm}$ gap at $V_{\mathrm{P}}=60 \mathrm{kV}$, the C-supply delivers $10 \mathrm{~A}$ and the TLT-supply the very similar value of $13 \mathrm{~A}$. Furthermore the streamer patterns in these two cases are quite similar as figures 5 and 8(a) show. We conclude that power supplies will create similar streamer patterns if their voltage rise time, peak voltage and internal resistance are similar, and that the internal resistance plays a decisive role.

\subsection{Thick and thin streamers, streamer branching}

For ease of discussion, streamers of different widths are characterized here as four different 'types', though they do not seem to be distinguished by phase transitions or different propagation modes.

- Type 1 streamers are very thick with a diameter of about $2.5 \mathrm{~mm}$; their velocity is just over $1 \mathrm{~mm} \mathrm{~ns}^{-1}$ and they carry currents of up to $25 \mathrm{~A}$.

- Type 2 streamers are thick with a diameter of about $1.2 \mathrm{~mm}$, a velocity of $0.5 \mathrm{~mm} \mathrm{~ns}^{-1}$ and currents of the order of $1 \mathrm{~A}$.

- Type 3 streamers are thin; their diameter is $0.2 \mathrm{~mm}$ which can only be properly determined by zooming in sufficiently with the camera (cf table 1 ), their velocity is $\sim 0.1 \mathrm{~mm} \mathrm{~ns}^{-1}$ and their current $\sim 10 \mathrm{~mA}$.

- Type 4 streamers are late; they start to propagate after streamers of type 1 or 2 have crossed the gap and their diameter appears to be similar to type 3 streamers. Their velocity and current could not be determined but are expected again to be similar to type 3 streamers. Type 4 streamers occasionally connect to the already existing streamer paths of type 1 or 2 .

A qualitative explanation of these different streamer diameters is that high local electric fields, in particular, fields that exceed the breakdown threshold [33] close to the needle electrode, create wide streamers. Similar observations of increasing negative streamer diameters in increasing fields can be found in the simulations presented in $[16,23,24]$. On the other hand, if the voltage rise time is comparable to streamer formation and propagation time, streamers can initiate near the needle while the local field is still lower, and the streamers are then more narrow. Therefore one could expect that a voltage rise time of $10 \mathrm{~ns}$ or less for a $60 \mathrm{kV}$ voltage pulse would create even thicker streamers. This is consistent with streamers of $10 \mathrm{~mm}$ diameter in [9] that are created in a larger $\mathrm{d} V / \mathrm{d} t$ (pulses of $140 \mathrm{kV}$ with $30 \mathrm{~ns}$ rise time) in a wire-cylinder gap of $145 \mathrm{~mm}$ radius.

In the present point-plane electrode geometry, streamers approximately keep their initial diameter between branching events, though recently in a wire-plate electrode geometry streamer diameters were observed to grow [34]. The streamers characterized as type 3 and 4 might be streamers of minimal diameter. Such a minimal diameter is necessary for the specific mode of streamer propagation by local field enhancement [3]; it requires the streamer diameter to be larger than the thickness of the charged ionization front in the streamer head [3].

In contrast to these streamers of probably minimal diameter ('minimal streamers'), the thick streamers of type 1 and 2 propagate faster and in higher background fields. They seem to come with a continuous variety of diameters. It is surprising to note that the average distance between branching is much larger for thick streamers than for thin streamers. An explanation of this fact is presently not at hand.

Another intriguing phenomenon is that late (type 4) streamers can bend towards earlier channels as shown, e.g. in figure $6(b)$. Similar phenomena have recently been observed in sprites [32]. However, in the present setup, it is likely that the streamer channels change polarity after reaching the electrode and therefore attract later streamers; this mechanism is unlikely for sprites in high layers of the atmosphere.

\subsection{An estimate on the current density}

We observe that the current density in streamers seems to be rather unchanged in quite different streamer patterns generated by different circuits. The estimate is based on the fact that the peak current lasts about as long as the streamers propagate.

The highest current peak measured is $48 \mathrm{~A}$ in figure $7(b)$. When one assumes that this current initially near the electrode is carried by only two thick streamers with a diameter of $5 \mathrm{~mm}$, the current density in such a streamer is $\sim 1.2 \mathrm{~A} \mathrm{~mm}^{-2}$. Looking further down in the same figure, 12 streamers of type 2 can be identified. With a diameter of $1.2 \mathrm{~mm}$ this gives a current density of $\sim 3.5 \mathrm{~A} \mathrm{~mm}^{-2}$. Figure $8(b)$ shows the measurement with the largest number of thin streamers, namely more than 200. Here the current is $3 \mathrm{~A}$ in total, i.e. $\sim 0.015 \mathrm{~A}$ per streamer. The diameter of this type 3 streamer is $0.2 \mathrm{~mm}$, therefore the current density is $0.5 \mathrm{~A} \mathrm{~mm}^{-2}$.

According to this very rough estimate, the current density within the streamer varies by a factor of $\sim 7$ while the current within a single streamer varies by a factor of $\sim 1600$.

\subsection{Final remarks and theoretical challenges}

Pulsed positive corona discharges in air show a large variety of streamer diameters and consecutive branching patterns. We suggest that this is determined by three properties of the external electric circuit: the peak voltage, the voltage rise time and the internal resistance.

We have presented our experimental results. It appears that all streamer photographs known to us fit in the presented frame of different diameters under the indicated conditions, such as gap size and pulse parameters. Obviously they ask for a theoretical explanation: can theory reproduce streamer diameters, current, current density and velocity as a function of the external circuit? Which additional insight does theory give into the inner structure of the streamer that is experimentally not accessible? The recent progress in numerical simulations of streamer dynamics, e.g. in [8,16,20,22-24], appears to make it possible to address the questions raised here in the near future.

A detailed understanding of the generated streamer structures requires us to distinguish different stages of the dynamics, namely (i) the inception and nucleation process next to the pointed anode, (ii) the streamer propagation and branching dynamics and (iii) the late stages of evolution after the first streamers have crossed the gap. These are clearly distinct processes and should be analysed separately.

(i) The first step is the streamer inception. Our experiments show that a fast rise to a high voltage together with a 
low impedance of the circuit favour the formation of thick streamers next to the pointed electrode; these streamers are fast and can carry a high current. For the formation of these streamers, the source of free electrons and the anode processes in the instantaneous local field need to be modelled appropriately. An ionization seed is formed in the high field region around the pointed electrode that then propagates outwards and destabilizes into a number of streamer branches. This nucleation process determines the number and size of the emerging streamers.

(ii) In the second phase each streamer carries a given charge, enhances the local field and propagates outwards. Here the challenge lies in understanding the diameter, velocity and branching process of a streamer head that is characterized by a certain head potential and charge content. We stress our conviction [3] that the electric potential alone is an insufficient characterization, and that different streamer diameters need to be related to different amounts of electric charge carried by the respective streamer heads. The splitting of one thick into several thinner streamers is then understood also as a splitting of the charge content of the original streamer over several new streamers-where we emphasize that electric charge is a conserved physical quantity.

(iii) After the streamer has reached the cathode, return stroke and electric recharging determine the further current flow and electric interaction of the channel.

We conclude by remarking that recently it was shown that the thick streamers created by very short pulses are very efficient for ozone production $[34,35]$. They also use the power supply in the most efficient way [29]. The present study therefore not only increases our fundamental knowledge on streamer discharges, but is also very relevant for applications.

\section{Acknowledgments}

This work is financially supported by STW under Contract Number CMM 6501 and by NWO under Contract Number 047.016.017.

\section{References}

[1] Raether H 1939 Z. Phys. 112464

[2] Meek J M and Craggs J D 1953 Electrical Breakdown of Gases (Oxford: Clarendon)

[3] Ebert U, Montijn C, Briels T M P, Hundsdorfer W, Meulenbroek B, Rocco A and van Veldhuizen E M 2006 Plasma Sources Sci. Technol. 15 S118

[4] Waters R T and Jones R E 1964 Phil. Trans. R. Soc. Lond. Ser. A 256185

[5] van Veldhuizen E M (ed) 2000 Electrical Discharges for Environmental Purposes: Fundamentals and Applications (Huntington, NY: Nova Science) ISBN 1-56072-743-8, pp 420

[6] Bastien F and Marode E 1979 J. Phys. D: Appl. Phys. 12249

[7] Creyghton Y L M, van Veldhuizen E M and Rutgers W R 1993 Electrical and optical study of pulsed positive corona
Non-Thermal Plasmas for Pollution Control (NATO ASI Series, subseries $G$, vol 34) ed B M Penetrante and S E Schultheis (Berlin: Springer) pp 205-30 (part A)

[8] Pancheshnyi S V, Nudovna M and Starikovskii A Y 2005 Phys. Rev. E 71016407

[9] Blom P P M, Smit C, Lemmens R H P and van Heesch E J M 1994 Combined optical and electrical measurements on pulsed corona discharges Gaseous Dielectrics vol VII, ed L G Christophorou and D R James (New York: Plenum)

[10] Grabowski L R, Briels T M P, van Veldhuizen E M and Pemen A J M 2005 Streamers in pulsed positive corona: low and high current regimes 17th ICPIG (Eindhoven, the Netherlands) file 04-425 available on www.icpig2005.nl

[11] Sentman D D, Wescott E M, Osborne D L and Heavner M J 1995 Geophys. Res. Lett. 221205

[12] Pasko V P, Stanley M A, Mathews J D, Inan U S and Wood T G 2002 Nature 416152

[13] Gerken E A, Inan U S and Barrington-Leigh C P 2002 Geophys. Res. Lett. 2982

[14] Zheleznyak M, Mnatsakanyan A and Sizykh S 1982 High Temp. 20357

[15] Liu N and Pasko V P 2004 J. Geophys. Res. 109 A04301

[16] Luque A, Ebert U, Montijn C and Hundsdorfer W 2006 Photoionization in negative streamers: fast computations and two propagation modes Preprint physics/0609247

[17] Dawson G A and Winn W P 1965 Z. Phys. 183159

[18] D'yakonov M I and Kachorovskii V Yu 1988 Sov. Phys._JETP 671049

[19] Raizer Yu P and Simakov A N 1998 Plasma Phys. Rep. 24700

[20] Liu N and Pasko V P 2006 J. Phys. D: Appl. Phys. 39327

[21] Kulikovsky A A 1998 Phys. Rev. E 577066

[22] Pancheshnyi S V and Starikovskii A Y 2003 J. Phys. D: Appl. Phys. 362683

[23] Montijn C, Ebert U and Hundsdorfer W 2006 Phys. Rev. E $73065401(\mathrm{R})$

[24] Montijn C, Hundsdorfer W and Ebert U 2006 J. Comput. Phys. 219801

[25] Arrayás M, Ebert U and Hundsdorfer W 2002 Phys. Rev. Lett. 88174502

[26] Rocco A, Ebert U and Hundsdorfer W 2002 Phys. Rev. E 66035102

[27] van Veldhuizen E M and Rutgers W R 2002 J. Phys. D: Appl. Phys. 352169

[28] Gravendeel B 1987 PhD Thesis Eindhoven University Technology, the Netherlands

[29] Yan K, van Heesch E J M, Pemen A J M, Huijbrechts P A H J and van der Laan P C T 2001 Rev. Sci. Instrum. 722443

[30] Briels T M P, van Veldhuizen E M and Ebert U 2005 Experiments on the diameter of positive streamers in air 17th ICPIG (Eindhoven, The Netherlands) file 04-418, available on www.icpig2005.nl

[31] van Veldhuizen E M, Kemps P C M and Rutgers W R 2002 IEEE Trans. Plasma Sci. 30162

[32] Cummer S A, Jaugey N, Li J, Lyons W A, Nelson T E and Gerken E A 2006 Geophys. Res. Lett. 33 L04104

[33] Montijn C and Ebert U 2006 J. Phys. D: Appl. Phys. 392979

[34] Winands G J J, Liu Z, Pemen A J M, van Heesch E J M, Yan K and van Veldhuizen E M 2006 J. Phys. D: Appl. Phys. 393010

[35] Grabowski L R, van Veldhuizen E M, Pemen A J M and Rutgers W R 2006 Plasma Chem. Plasma Process. 263 